\title{
Prostate Carcinogenesis with Diabetes and Androgen-Deprivation-Therapy-Related Diabetes: An Update
}

\author{
Noboru Hara \\ Division of Urology, Department of Regenerative and Transplant Medicine, and Division of Molecular Oncology, \\ Department of Signal Transduction Research, Graduate School of Medical and Dental Sciences, Niigata University, \\ Asahimachi 1, Niigata 951-8510, Japan \\ Correspondence should be addressed to Noboru Hara, harasho@med.niigata-u.ac.jp
}

Received 14 December 2011; Accepted 10 May 2012

Academic Editor: Joseph R. Landolph Jr.

Copyright ( 2012 Noboru Hara. This is an open access article distributed under the Creative Commons Attribution License, which permits unrestricted use, distribution, and reproduction in any medium, provided the original work is properly cited.

\begin{abstract}
Prostate cancer and the androgen deprivation therapy (ADT) thereof are involved in diabetes in terms of diabetes-associated carcinogenesis and ADT-related metabolic disorder, respectively. The aim of this study is to systematically review relevant literature. About 218,000 men are estimated to be newly diagnosed with prostate cancer every year in the United States. Approximately $10 \%$ of them are still found with metastasis, and in addition to them, about $30 \%$ of patients with nonmetastatic prostate cancer recently experience ADT. Population-based studies have shown that dissimilar to other malignancies, type 2 diabetes is associated with a lower incidence of prostate cancer, whereas recent large cohort studies have reported the association of diabetes with advanced high-grade prostate cancer. Although the reason for the lower prevalence of prostate cancer among diabetic men remains unknown, the lower serum testosterone and PSA levels in them can account for the increased risk of advanced disease at diagnosis. Meanwhile, insulin resistance already appears in $25-60 \%$ of the patients 3 months after the introduction of ADT, and long-term ADT leads to a higher incidence of diabetes (reported hazard ratio of 1.28-1.44). Although the possible relevance of cytokines such as Il-6 and TNF- $\alpha$ to ADT-related diabetes has been suggested, its mechanism is poorly understood.
\end{abstract}

\section{Introduction}

Prostate cancer and the hormonal therapy thereof (androgen deprivation therapy, $\mathrm{ADT}$ ) have been associated with diabetes in terms of diabetes-associated carcinogenesis [1] and ADT-related metabolic disorder [2], respectively. The present paper systematically introduces prostate carcinogenesis with diabetes and ADT-related diabetes/insulin resistance both in epidemiological and etiological approaches.

\section{Search Method}

PubMed and MEDLINE searches were performed for articles published between January 1991 and November 2011 based on the following key words for diabetes-associated prostate carcinogenesis: prostate cancer AND insulin resistance, hyperglycemia, cancer risk, and diabetes. Literature on ADT-related diabetes was searched using the following keywords: androgen deprivation therapy OR hormone therapy AND diabetes, insulin resistance, hyperglycemia, and metabolic syndrome. Relevant articles on growth hormone (GH)/insulin-like growth factor (IGF)-1 and androgen metabolism were searched with similar strategy. Except for studies concerning statistics, meta-analysis, or reanalysis, review articles were excluded. All full papers based on evidence level 1 and 2 and full papers on level 3 supporting them were downloaded via the library of our institution, provided from other institutions, or purchased, and relevant articles on experimental studies were obtained by similar methods.

\section{General Statistics of Prostate Cancer}

Prostate cancer is a common malignancy around the world, and in the United States, about 218,000 and 32,000 men are estimated to be newly diagnosed with and to die of prostate cancer every year, respectively [3]. Therapeutic options 
for prostate cancer are determined with informed-consent according to the disease-specific risk and patient's conditions such as age and comorbidities. Although the prostate-specific antigen (PSA) test has led to a stage migration with increased low- to intermediate-risk localized disease, about $10 \%$ of the patients are still found with metastatic disease at diagnosis [4]. Additionally, 20-35\% of the patients are categorized as having locally advanced disease or localized high-risk cancer based on high histopathological grade (Gleason score of 810 ) or high PSA level (serum PSA higher than $20 \mathrm{ng} / \mathrm{mL}$ ) $[5,6]$.

\section{The Presence of Diabetes and the Incidence of Prostate Cancer}

Large cohort studies have shown that diabetes is associated with a higher incidence of many malignancies including lung, gastric, colorectal, liver, and pancreatic cancer [7, 8]. Several molecular mechanisms have been suggested for their association, for example, insulin resistance leading to high cell proliferation by the activation of the phosphatidylinositol 3-kinase/Akt/mammalian target of rapamycin pathway, elevated leptin/adiponectin linking to impaired anticancer immunity, and upregulated inflammation/tumor necrosis factor-alpha (TNF- $\alpha$ ) leading to cancer cell survival; they produce a complex network with many-to-many correspondence $[9,10]$. Conversely, the mentioned cohort studies reported a lower prevalence of prostate cancer among men with type 2 diabetes compared with that in men without diabetes. Recently, Turner and colleagues reported that diabetes was associated with a reduced risk of prostate cancer (odds ratio $=0.78 ; 95 \%$ CI: $0.61-0.99$ ) [11], and a study referring to the Swedish national database and nationwide Cancer Registry also showed a lower risk of prostate cancer in a total of 125,126 registered type 2 diabetes men [12]. Most recently, Atchison and associates reported that men with diabetes had a decreased risk of prostate cancer $(\mathrm{RR}=0.89,95 \% \mathrm{CI}=0.87-0.91)$ [7]. These results imply a specific relationship between diabetes and prostate cancer; however, it remains unknown why type 2 diabetes is associated with a lower incidence of prostate cancer. In a retrospective study enrolling 3,162 consecutive men who underwent prostate biopsy, Moses and associates showed that, though not significant, those with diabetes had higher odds of histologically more aggressive disease (Gleason score of 7 or higher) than those without diabetes (OR 1.31, 95\% CI: $0.98-1.74 ; P=0.07)$ [13]. In their study, diabetes also led to an increased risk of overall prostate cancer in the cohort (OR 1.26, 95\% CI: 1.01-1.55; $P=0.04$ ). It is suggested that the study design and cohort in the study by Moses et al. mainly comprising men with elevated PSA on prostate cancer screening possibly involves different patients' background.

It is known from the results of recent population-based cohort studies that men with type 2 diabetes show lower PSA levels than those without diabetes [14-16]. Considering evidence on reduced PSA levels in diabetic men, is exposure to PSA screening associated with a reduced risk of prostate cancer in men with diabetes? In a longitudinal observational study enrolling 4,511 men with newly diagnosed prostate cancer between 1986 and 2004, Kasper et al. demonstrated the increased risk of prostate cancer in diabetic men after PSA era compared with that in pre-PSA era, although the odds ratio still remained low after PSA era (0.86) [17]. In a recent population-based study conducted in Taiwan, 985,815 study subjects including 104,343 diabetes patients identified in 1997 were followed up between 1998 and 2009; the unadjusted and adjusted risk ratios in diabetes men for incident prostate cancer were 6.97 (5.34-9.10) $P<0.001$ and 1.56 (1.19-2.04) $P=0.0013$, respectively [18]. However, the rate of exposure to PSA screening in this population was unclear.

The relevance of lower PSA levels to the reduced risk of prostate cancer in men with diabetes is thus equivocal, but men with diabetes potentially have more advanced disease at diagnosis where their PSA level reaches a certain cut-off/threshold. Correspondingly, two recent large cohort studies reported the association of diabetes with high/poorrisk disease: more advanced clinical stage and higher Gleason sore [1]. In a cohort study, Li et al. reported that men with diabetes had a higher risk of advanced prostate cancer with a multivariate adjusted HR of 1.89 (95\% CI: 1.02-3.50) in 230 men with prostate cancer newly identified among 22,458 Japanese men [19]. Although retrospective, several large studies have also reported the relationship between diabetes and high-grade prostate cancer $[20,21]$.

Accordingly, diabetes is associated with a lower PSA level in the general population and a higher incidence of poor-risk prostate cancer in the screening-based cohort or regional cancer registration. The latter can be explained by the frequent reduced testosterone levels in men with increased insulin resistance or type 2 diabetes [22] and is concordant with previous study results on prostate cancer biology; low testosterone environment in vivo is involved in high Gleason score [23-25], advanced disease stages [26], and a poor prognosis [27, 28]. All of these studies have suggested that the adaptation of cancer cells to lowtestosterone milieu links to their high viability and malignant potential. Most recently, Botto and associates reported a high incidence of predominant Gleason pattern 4 (histologically high-grade pattern) in men with prostate cancer and low serum testosterone [29]. They performed a prospective study on 452 men who underwent radical prostatectomy; the final study group comprised 431 eligible patients. In surgical specimens, 132 patients (31\%) had predominant Gleason pattern 4 , and their serum total testosterone level was lower than that in the remaining 299 with predominant lower histological grade ( 4.00 versus $4.50 \mathrm{ng} / \mathrm{mL}, P=0.001)$. In men with predominant Gleason pattern 4 , interestingly, the diabetes history was noted more frequently $(8.4 \%$ versus $2.7 \%, P=0.008)$. Accordingly, diabetes is involved in the incidence of high-grade/advanced prostate cancer most probably via the acquisition of more malignant potential under low-testosterone environment.

Meanwhile, the mechanism of lower PSA levels in diabetic men is hard to explain; it is still unclear why diabetes is associated with lower PSA levels. As described elsewhere, serum testosterone levels in men with type 2 
diabetes are likely to be lower [22]. Yet, their deference from the normo-gonadotropic testosterone level is about $30 \%$ in median. It remains unknown whether the decrease of testosterone levels in such degree has an impact on serum PSA levels. Morgentaler advocated a theory that can account for such contradiction between androgen and PSA levels; there is a limit to the ability of testosterone to stimulate androgenic activities including prostate epithelium proliferation [30]. The Saturation Model explains the observation that prostate epithelium proliferation is testosterone dependent in serum testosterone concentrations at or below the near-castrate level (levels of $95 \%$ or more testosterone being deprived) and becomes testosterone-independent above this concentration. Physiologic concentrations of testosterone provide an excess of testosterone and its intracellular prostatic metabolite dihydrotestosterone, which maintains optimal prostatic growth. Reducing testosterone concentration below a critical concentration threshold (the Saturation Point) leads to an intracellular milieu where prostate tissue grows in an androgen-dependent manner $[25,30,31]$. Thus, the mild decrease of testosterone levels in diabetic men does not seemingly explicate their lower serum PSA level.

Another interest is whether a higher insulin level is associated with a higher incidence of prostate cancer. Stocks and colleagues prospectively performed conditional logistic regression analyses on 392 prostate cancer patients and 392 matched controls [32]. In their study, homeostatic model assessment of insulin resistance (HOMA-IR) was lower in the prostate cancer group than in the control group $(1.5 \pm 0.7$ versus $1.6 \pm 0.7)$, and the increasing level of HOMA-IR was associated with the decrease in risk of prostate cancer (Odds ratio $=0.60,95 \% \mathrm{CI}, 0.38-0.94, P=0.03)$. In another casecontrol study by Chen et al. with 174 men in each of the case and control groups, insulin levels had no impact on the risk of incident prostate cancer [33]. In contrast, a recent cohort study with 9-year observation by Hammarsten and associates showed that the prediagnostic insulin level was higher in men with than without incident prostate cancer (fasting serum insulin 12.0 versus $9.0 \mathrm{mU} / \mathrm{l}, P=0.023$ ), although the study included a small number of prostate cancer patients $(n=44)$ and hazard ratio for the insulin level was unclear [34]. These varied results may possibly depend on study designs and length of the observation period. In a recent case-cohort study on a large registered cohort, Albanes et al. reported that increased insulin levels were associated with increased risks of prostate cancer $(\mathrm{OR}=1.50-2.55$ among compared insulin quartiles, $P=0.02$ ) [35]. Another previous populationbased study reported similar results [36].

In etiological approaches, the regulation and metabolism of insulin and IGF-1 are correlated, sharing homologous molecular structures [37], while many studies have shown the impact of high circulating IGF-1 levels on prostate carcinogenesis $[38,39]$. This was also established experimentally before PSA era [40]. However, a recent large prospective study as well as previous studies has concluded the absence of correlation between the plasma IGF-1 level and insulin resistance [32]. In genetics, some reported no association between type 2 diabetes risk variants and prostate cancer risk
[41], whereas some suggested a possible [42] or inverse [43] association between them.

Most recently, an experimental study reported intracellular de novo steroidogenesis promoted by insulin in prostate cancer; Lubik et al. showed that transcription of androgenmetabolic enzymes such as CYP17A1 and 5 - $\alpha$-reductase were upregulated by insulin in a dose-dependent manner in prostate cancer cells LNCaP and 22RV1, which express androgen receptor [44]. In their study, the protein level of CYP17A1 in LNCaP also increased significantly with insulin, and the intracellular level of dehydroepiandrosterone and testosterone increased 18 -fold and 60 -fold by insulin, respectively, $(P<0.05$ in both $)$ with PSA secretion increased significantly. These results suggest that insulin may directly promote proliferation of prostate cancer cells. However, these observations are based on an experimental model for castration-resistant prostate cancer, and studies to examine the effect of insulin on prostate tumorigenesis during its early phase or in hormone-naïve cancer are needed.

Thus, the relationship among insulin resistance, testosterone milieu, PSA level, incidence of prostate cancer, and its malignant potential in men with diabetes has not been fully elucidated, and remains a matter of concern for the regulation of prostate carcinogenesis as well as advances in management of prostate cancer in the general population. Further studies are required in both experimental and clinical approaches.

\section{Practice of ADT}

Both benign and malignant prostatic epithelial cells are well known to receive proliferative stimuli from androgens and to have androgen-dependent bioactivities, and ADT has been the therapeutic mainstay for men with metastasis or recurrent disease following definitive local therapy, although the treatment effect is palliative in most of the former [45]. $\mathrm{ADT}$ is performed with surgical castration or injection of gonadotropin-releasing hormone $(\mathrm{GnRH})$ analogues with or without peroral antiandrogens. In 90s, the use of ADT rapidly increased from a small percent to $30 \%$ in the United States [46], and ADT has recently been used in about $30 \%$ of patients with localized or locally advanced prostate cancer, mainly combined with radiotherapy for intermediate- to high-risk disease [47]. It is estimated that more than 600,000 men receive $\mathrm{ADT}$ and that one in two prostate cancer patients experiences ADT in some treatment setting in the United States [48], whereas annual claims for $\mathrm{GnRH}$ analogues decreased by $25.1 \%$ and $16.8 \%$ from 2004 to 2007 in the Medicare and the Veterans Health Administration populations, respectively, most probably due to prevailing intermittent $\mathrm{ADT}$ and expectant management policy in increasing awareness about ADT-related adverse effects [49].

\section{ADT-Related Insulin Resistance and Diabetes}

It is estimated that the 5-year disease-specific survival for men with prostate cancer reaches $98 \%[3,6]$. In particular, 
men with localized prostate cancer almost exclusively die of other causes, and causes of death in them are similar to those of the general male population [50]. Therefore, ADT-related toxicity and the management thereof are critical in clinical practice.

As discussed elsewhere, reduced testosterone levels are associated with insulin resistance and type 2 diabetes in the general population [22]. Insulin resistance appears early during ADT; some previous prospective studies showed that increased fasting insulin levels already emerge in 26-63\% of the patients 3 months after the inception of ADT [51, 52]. Hyperinsulinemia during the early period of ADT possibly counteracts against the development of diabetes. Yet, longterm ADT leads to a higher incidence of diabetes as shown in following large population-based studies, although there has been no prospective longitudinal study with a long observation period.

Keating et al. used Surveillance, Epidemiology, and End Results (SEER) Medicare data; the study cohort comprised 73,196 men with localized prostate cancer [53]. Among the 64,721 men without prevalent diabetes, 10.9\% developed diabetes, and its adjusted hazard ratio was 1.44 (95\% CI: 1.34 to $1.55, P<0.001)$ in men treated with $\mathrm{GnRH}$ agonists. The same authors most recently performed another large population-based study and reported an increased risk of incident diabetes in men undergoing ADT with GnRH agonists (adjusted hazard ratio: $1.28,95 \% \mathrm{CI}: 1.19$ to $1.38, P<$ 0.001) [2]. A Canadian population-based study also showed an increased incidence of diabetes in men treated with $\mathrm{GnRH}$ agonists (HR: 1.16, 95\% CI: 1.11-1.21, $P<0.001$ ) [54]. Another large study, though retrospectively, reported that $8.94 \%$ of men who were treated with ADT $(n=1,231)$ were diagnosed with diabetes 12 months after ADT, while 6.99\% of those without ADT $(n=7,250)(P=0.02)$ [55]. Thus, this evidence strongly supports the demand of large welldesigned studies that longitudinally analyze the incidence of ADT-related metabolic disorders with long-term followup. Moreover, the pretreatment evaluation and posttreatment followup for diabetes and the relevant conditions are possibly important to improve overall survival in men receiving ADT. However, there has been no interventional study to determine appropriate/efficient screening methods and follow-up interval.

On the other hand, a few trials examined the effect of exercise, diet, and supportive agents/supplements in men during ADT. Nobes et al. reported the efficacy of a low glycemic index diet, exercise program, and metformin ( $850 \mathrm{mg}$ daily to $850 \mathrm{mg}$ twice daily) in men treated with GnRH agonist (6-month ADT) in a prospective randomized study [56]. The intervention $\operatorname{arm}(n=20)$ had a reduction in abdominal girth $(P=0.05)$, weight $(P<0.001)$, and body mass index $(P<0.001)$ compared to controls $(n=20)$. Although the study was designed in a small pilot volume, changes in biochemical markers of insulin resistance did not differ between the two arms during the study. Lebret and colleagues examined the utility of an educational toolkit consisting of information brochure concerning adverse effects of ADT, practical guidance on lifestyle, recipe booklet for ADT-adapted diet, and lifestyle diary to record and evaluate the life style and body measurement [57]. They recruited more than 500 men with prostate cancer receiving ADT, but the aim of the study was to test a tool-kit designed to improve well-being in patients with prostate cancer, and relevant studies on its impact on metabolic disorder during ADT are warranted.

The etiology of ADT-related diabetes is poorly understood. As mentioned above, increased fasting insulin levels are observed early after the initiation of ADT, suggesting possible primary responses to altered hormonal milieu. Additionally, recent studies showed that 6-month ADT with combined $\mathrm{GnRH}$ agonist and antiandrogens is associated with an about $10 \%$ increase of serum IGF-1 [5861]. Although evidence supporting that low testosterone environment directly brings about the increased insulin level is absent, several previous studies have suggested associations among diabetes, cytokines, and sex steroid levels. Proinflammatory cytokines such as interleukin-6 (IL6 ), and TNF- $\alpha$ secreted by macrophages and monocytes in response to infection play a critical role in immunity. Type 2 diabetes has been involved in innate immune system disorder with chronic low-grade inflammation $[62,63]$, and many studies have shown that serum/plasma levels of inflammatory markers represented by TNF- $\alpha$ and IL- 6 in patients with elevated fasting blood glucose are independent values predictive of development of diabetes, thereby adipose tissue being the major source of these cytokines $[64,65]$. Relevance of elevated serum IL- 6 , and TNF- $\alpha$ levels to insulin resistance and diabetes has been shown accordingly.

Besides, sex steroids such as 17beta-estradiol (estradiol) and testosterone have been suggested to play a role in modulating inflammation, although relevant studies are limited. A previous study showed that estradiol withdrawal brought about greater expressions of proinflammatory cytokines represented by IL- 6 and TNF- $\alpha$ in human monocyte-derived macrophages of premenopausal women [66]. Concerning androgens, an in vitro study showed inhibition of IL- 6 mRNA transcription and TNF release by dihydrotestosterone [67]. Some clinical trials have shown the influence of testosterone administration on cytokines or inflammation. In the doubleblinded placebo-controlled crossover study on 20 hypogonadal type 2 diabetic men by Kapoor et al, although testosterone treatment reduced leptin $(-7141.9 \pm 1461.8 \mathrm{pg} / \mathrm{mL}$; $P=0.0001)$ and adiponectin levels $(-2075.8 \pm 852.3 \mathrm{ng} / \mathrm{mL}$; $P=0.02)$, its effect on the TNF- $\alpha$, IL-6 or CRP level was not significant [68]; the small study volume may possibly lead to a negative result on cytokines.

Most recently, Kalinchenko and associates studied the effect of testosterone replacement on diabetic and inflammatory markers in 184 men with metabolic syndrome and hypogonadism in a randomized, placebo-controlled, doubleblinded setting [69]. In the testosterone-treated group, plasma insulin and HOMA-IR decreased compared with those in the placebo-treated group $(P=0.07$ and 0.04 , resp.). Thereby, TNF- $\alpha$ and CRP of the testosterone-treated group declined 30 weeks after treatment compared to those at baseline $(19 \mathrm{mg} / \mathrm{dL}$ versus $29 \mathrm{mg} / \mathrm{L}, P<0.001$ compared to control and $2.4 \mathrm{ng} / \mathrm{l}$ versus $3.5 \mathrm{ng} / \mathrm{l}, P=0.03$ compared to control, resp.); however, IL-6 levels were equivalent 
between before and after treatment ( $1.1 \mathrm{ng} / \mathrm{l}$ versus $1.1 \mathrm{ng} / \mathrm{l})$. Additionally, subcutaneous abdominal fat has been shown to be an important index reflecting insulin resistance and relevant inflammation. A recent study focused on increased HOMA-IR $(2.50 \pm 1.12$ to $2.79 \pm 1.31, P<0.05)$ and subcutaneous abdominal fat area $240.7 \pm 107.5$ to $271.3 \pm$ $92.8 \mathrm{~cm}^{2}, P<0.01$ ) [70], while abdominal fat mass has been associated with insulin resistance and the innate immune activation [71].

Regarding the effect of ADT on circulating proinflammatory cytokines, the relevant study has barely been presented. A recent study prospectively examined the relationship between these cytokines and sex steroid levels in the serum in 72 men with localized prostate cancer, who received ADT with GnRH agonists [72]. The authors reported an altered association of interleukin- 6 with sex steroids during ADT as follows: before ADT, similar to the previous reports, serum interleukin-6 levels were inversely correlated with serum total-testosterone (Spearman's rank correlation coefficient rs $=-0.305, P=0.009$ ) and dihydrotestosterone ( $\mathrm{rs}=$ $-0.308, P=0.006)$ concentrations, but not correlated with adrenal androgen or estradiol levels. After ADT, in contrast to the pretreatment relationship, interleukin-6 levels were positively correlated with total-testosterone concentrations (rs $=0.343, P=0.003$ ), and were positively correlated also with levels of androstenedione ( $\mathrm{rs}=0.351, P=0.002)$ and estradiol ( $\mathrm{rs}=0.335, P=0.004$ ), suggesting a coordinated regulation emerging between proinflammatory cytokines and sex steroids during ADT. Although the study focusing on ADT-related body composition change concluded the unchanged IL-6 level despite increased \%body fat, the alteration of hormonal milieu produced by ADT can theoretically have an influence on the association of proinflammatory cytokines with metabolic activities including insulin sensitivity. However, it remains unclear whether such altered association between sex steroids and proinflammatory cytokines is the primary action with insulin resistance or secondary reaction to reduced testosterone levels, and further studies are warranted to elucidate the mechanism of ADT-related diabetes and to overcome this important adverse effect brought about by ADT.

\section{Conclusion}

Diabetes is associated with a lower PSA level in the general population and a higher incidence of advanced prostate cancer in the prostate cancer registration-based cohort. Although the mechanism of the former association is unknown, the latter can be explained by reduced testosterone levels in men with increased insulin resistance or type 2 diabetes. Insulin resistance is frequently observed early after the introduction of ADT, and long-term ADT links to the increased risk of development of diabetes. However, the mechanism of ADT-related diabetes remains unclear; a regulatory relationship between proinflammatory cytokines and sex steroids is possibly involved in ADT-related diabetes.

\section{Abbreviations}

PSA: $\quad$ Prostate-specific antigen

ADT: Androgen deprivation therapy

GnRH: Gonadotropin-releasing hormone

HOMA-IR: Homeostatic model assessment of insulin resistance.

\section{Conflict of Interests}

The author declares no conflict of interests.

\section{Acknowledgment}

This work was supported in part by a Grant-in-Aid-for Scientific Research from the Ministry of Education, Culture, Sports, Science and Technology, Japan (no. 21791493).

\section{References}

[1] C. Wu, D. M. Moreira, L. Gerber, R. S. Rittmaster, G. L. Andriole, and S. J. Freedland, "Diabetes and prostate cancer risk in the REDUCE trial," Prostate Cancer and Prostatic Diseases, vol. 14, no. 4, pp. 326-331, 2011.

[2] N. L. Keating, A. J. O’Malley, S. J. Freedland, and M. R. Smith, "Diabetes and cardiovascular disease during androgen deprivation therapy: observational study of veterans with prostate cancer," Journal of the National Cancer Institute, vol. 102, no. 1, pp. 39-46, 2010.

[3] A. Jemal, R. Siegel, J. Xu, and E. Ward, "Cancer statistics, 2010," CA Cancer Journal for Clinicians, vol. 60, no. 5, pp. 277$300,2010$.

[4] S. P. Elliott, S. L. Jarosek, T. J. Wilt, and B. A. Virnig, "Reduction in physician reimbursement and use of hormone therapy in prostate cancer," Journal of the National Cancer Institute, vol. 102, no. 24, pp. 1826-1834, 2010.

[5] M. R. Cooperberg, J. Cowan, J. M. Broering, and P. R. Carroll, "High-risk prostate cancer in the United States, 1990-2007," World Journal of Urology, vol. 26, no. 3, pp. 211-218, 2008.

[6] M. V. Meng, E. P. Elkin, D. M. Latini, J. DuChane, and P. R. Carroll, "Treatment of patients with high risk localized prostate cancer: results from Cancer of the Prostate Strategic Urological Research Endeavor (CaPSURE)," Journal of Urology, vol. 173, no. 5, pp. 1557-1561, 2005.

[7] E. A. Atchison, G. Gridley, J. D. Carreon, M. F. Leitzmann, and K. A. McGlynn, "Risk of cancer in a large cohort of U.S. veterans with diabetes," International Journal of Cancer, vol. 128, no. 3, pp. 635-643, 2011.

[8] B. Carstensen, D. R. Witte, and S. Friis, "Cancer occurrence in Danish diabetic patients: duration and insulin effects," Diabetologia, vol. 55, no. 4, pp. 948-958, 2012.

[9] E. J. Gallagher, Y. Fierz, A. Vijayakumar, N. Haddad, S. Yakar, and D. Leroith, "Inhibiting PI3K reduces mammary tumor growth and induces hyperglycemia in a mouse model of insulin resistance and hyperinsulinemia," Oncogene. In press.

[10] J. H. Egberts, V. Cloosters, A. Noack et al., "Anti-tumor necrosis factor therapy inhibits pancreatic tumor growth and metastasis," Cancer Research, vol. 68, no. 5, pp. 1443-1450, 2008.

[11] E. L. Turner, J. A. Lane, J. L. Donovan et al., "Association of diabetes mellitus with prostate cancer: nested case-control 
study (Prostate testing for cancer and Treatment study)," International Journal of Cancer, vol. 128, no. 2, pp. 440-446, 2011.

[12] K. Hemminki, X. Li, J. Sundquist, and K. Sundquist, "Risk of cancer following hospitalization for type 2 diabetes," Oncologist, vol. 15, no. 6, pp. 548-555, 2010.

[13] K. A. Moses, O. A. Utuama, M. Goodman, and M. M. Issa, "The association of diabetes and positive prostate biopsy in a US veteran population," Prostate Cancer and Prostatic Disease, vol. 15, no. 1, pp. 70-74, 2012.

[14] H. Müller, E. Raum, D. Rothenbacher, C. Stegmaier, and H. Brenner, "Association of diabetes and body mass index with levels of prostate-specific antigen: implications for correction of prostate-specific antigen cutoff values?" Cancer Epidemiology Biomarkers and Prevention, vol. 18, no. 5, pp. 1350-1356, 2009.

[15] L. P. Wallner, H. Morgenstern, M. E. McGree et al., "The effects of type 2 diabetes and hypertension on changes in serum prostate specific antigen levels: results from the olmsted county study," Urology, vol. 77, no. 1, pp. 137-141, 2011.

[16] K. M. Waters, B. E. Henderson, D. O. Stram, P. Wan, L. N. Kolonel, and C. A. Haiman, "Association of diabetes with prostate cancer risk in the multiethnic cohort," American Journal of Epidemiology, vol. 169, no. 8, pp. 937-945, 2009.

[17] J. S. Kasper, V. Liu, and E. Giovannucci, "Diabetes mellitus and risk of prostate cancer in the health professionals followup study," International Journal of Cancer, vol. 124, no. 6, pp. 1398-1403, 2009.

[18] M. Y. Lee, K. D. Lin, P. J. Hsiao, and S. J. Shin, "The association of diabetes mellitus with liver, colon, lung, and prostate cancer is independent of hypertension, hyperlipidemia, and gout in Taiwanese patients," Metabolism, vol. 61, no. 2, pp. 242-249, 2012.

[19] Q. Li, S. Kuriyama, M. Kakizaki et al., "History of diabetes mellitus and the risk of prostate cancer: the Ohsaki Cohort Study," Cancer Causes and Control, vol. 21, no. 7, pp. 10251032, 2010.

[20] D. M. Moreira, T. Anderson, L. Gerber et al., "The association of diabetes mellitus and high-grade prostate cancer in a multiethnic biopsy series," Cancer Causes and Control, vol. 22, no. 7, pp. 977-983, 2011.

[21] T. Mitin, M. H. Chen, Y. Zhang et al., "Diabetes mellitus, race and the odds of high grade prostate cancer in men treated with radiation therapy," The Journal of Urology, vol. 186, no. 6, pp. 2233-2238, 2011.

[22] M. Grossmann, M. C. Thomas, S. Panagiotopoulos et al., "Low testosterone levels are common and associated with insulin resistance in men with diabetes," Journal of Clinical Endocrinology and Metabolism, vol. 93, no. 5, pp. 1834-1840, 2008.

[23] M. A. Hoffman, W. C. DeWolf, and A. Morgentaler, "Is low serum free testosterone a marker for high grade prostate cancer?" Journal of Urology, vol. 163, no. 3, pp. 824-827, 2000.

[24] G. Schatzl, S. Madersbacher, T. Thurridl et al., "High-grade prostate cancer is associated with low serum testosterone levels," Prostate, vol. 47, no. 1, pp. 52-58, 2001.

[25] I. Takizawa, T. Nishiyama, N. Hara, E. Isahaya, T. Hoshii, and K. Takahashi, "Serum prostate-specific antigen levels reflect the androgen milieu in patients with localized prostate cancer receiving androgen deprivation therapy: tumor malignant potential and androgen milieu," Prostate, vol. 70, no. 13, pp. 1395-1401, 2010.
[26] J. C. Massengill, L. Sun, J. W. Moul et al., "Pretreatment total testosterone level predicts pathological stage in patients with localized prostate cancer treated with radical prostatectomy," Journal of Urology, vol. 169, no. 5, pp. 1670-1675, 2003.

[27] T. Imamoto, H. Suzuki, K. Akakura et al., "Pretreatment serum level of testosterone as a prognostic factor in Japanese men with hormonally treated stage D2 prostate cancer," Endocrine Journal, vol. 48, no. 5, pp. 573-578, 2001.

[28] G. W. Chodak, N. J. Vogelzang, R. J. Caplan et al., "Independent prognostic factors in patients with metastatic (stage D2) prostate cancer," Journal of the American Medical Association, vol. 265, no. 5, pp. 618-621, 1991.

[29] H. Botto, Y. Neuzillet, T. Lebret, P. Camparo, V. Molinie, and J. P. Raynaud, "High incidence of predominant Gleason pattern 4 localized prostate cancer is associated with low serum testosterone," The Journal of Urology, vol. 186, no. 4, pp. 1400-1405, 2011.

[30] A. Morgentaler, "Testosterone replacement therapy and prostate cancer," Urologic Clinics of North America, vol. 34, no. 4, pp. 555-563, 2007.

[31] A. Morgentaler and A. M. Traish, "Shifting the paradigm of testosterone and prostate cancer: the saturation model and the limits of androgen-dependent growth," European Urology, vol. 55, no. 2, pp. 310-320, 2009.

[32] T. Stocks, A. Lukanova, S. Rinaldi et al., "Insulin resistance is inversely related to prostate cancer: a prospective study in Northern Sweden," International Journal of Cancer, vol. 120, no. 12, pp. 2678-2686, 2007.

[33] C. Chen, S. K. Lewis, L. Voigt, A. Fitzpatrick, S. R. Plymate, and N. S. Weiss, "Prostate carcinoma incidence in relation to prediagnostic circulating levels of insulin-like growth factor I, insulin-like growth factor binding protein 3, and insulin," Cancer, vol. 103, no. 1, pp. 76-84, 2005.

[34] J. Hammarsten, J. E. Damber, R. Peeker, D. Mellström, and B. Högstedt, "A higher prediagnostic insulin level is a prospective risk factor for incident prostate cancer," Cancer Epidemiology, vol. 34, no. 5, pp. 574-579, 2010.

[35] D. Albanes, S. J. Weinstein, M. E. Wright et al., "Serum insulin, glucose, indices of insulin resistance, and risk of prostate cancer," Journal of the National Cancer Institute, vol. 101, no. 18, pp. 1272-1279, 2009.

[36] A. W. Hsing, Y. T. Gao, S. Chua, J. Deng, and F. Z. Stanczyk, "Insulin resistance and prostate cancer risk," Journal of the National Cancer Institute, vol. 95, no. 1, pp. 67-71, 2003.

[37] J. Boucher, Y. H. Tseng, and C. R. Kahn, "Insulin and insulinlike growth factor-1 receptors act as ligand-specific amplitude modulators of a common pathway regulating gene transcription," Journal of Biological Chemistry, vol. 285, no. 22, pp. 17235-17245, 2010.

[38] J. M. Chan, M. J. Stampfer, E. Giovannucci et al., "Plasma insulin-like growth factor-I and prostate cancer risk: a prospective study," Science, vol. 279, no. 5350, pp. 563-566, 1998.

[39] P. Stattin, S. Rinaldi, C. Biessy, U. H. Stenman, G. Hallmans, and R. Kaaks, "High levels of circulating insulin-like growth factor-I increase prostate cancer risk: a prospective study in a population-based nonscreened cohort," Journal of Clinical Oncology, vol. 22, no. 15, pp. 3104-3112, 2004.

[40] P. Cohen, D. M. Peehl, G. Lamson, and R. G. Rosenfeld, "Insulin-like growth factors (IGFs), IGF receptors, and IGFbinding proteins in primary cultures of prostate epithelial cells," Journal of Clinical Endocrinology and Metabolism, vol. 73, no. 2, pp. 401-407, 1991. 
[41] K. M. Waters, L. R. Wilkens, K. R. Monroe et al., "No association of type 2 diabetes risk variants and prostate cancer risk: the multiethnic cohort and PAGE," Cancer Epidemiology, Biomarkers \& Prevention, vol. 20, no. 9, pp. 1979-1981, 2011.

[42] V. L. Stevens, J. Ahn, J. Sun et al., "HNFIB and JAZFI genes, diabetes, and prostate cancer risk," Prostate, vol. 70, no. 6, pp. 601-607, 2010.

[43] T. E. Meyer, E. Boerwinkle, A. C. Morrison et al., "Diabetes genes and prostate cancer in the atherosclerosis risk in communities study," Cancer Epidemiology Biomarkers and Prevention, vol. 19, no. 2, pp. 558-565, 2010.

[44] A. A. Lubik, J. H. Gunter, S. C. Hendy et al., "Insulin increases de novo steroidogenesis in prostate cancer cells," Cancer Research, vol. 71, no. 17, pp. 5754-5756, 2011.

[45] T. Saito, N. Hara, Y. Kitamura, and S. Komatsubara, "Prostatespecific antigen/prostatic acid phosphatase ratio is significant prognostic factor in patients with stage IV prostate cancer," Urology, vol. 70, no. 4, pp. 702-705, 2007.

[46] V. B. Shahinian, Y. F. Kuo, J. L. Freeman, E. Orihuela, and J. S. Goodwin, "Increasing use of gonadotropin-releasing hormone agonists for the treatment of localized prostate carcinoma," Cancer, vol. 103, no. 8, pp. 1615-1624, 2005.

[47] S. M. Gilbert, Y. F. Kuo, and V. B. Shahinian, "Prevalent and incident use of androgen deprivation therapy among men with prostate cancer in the United States," Urologic Oncology, vol. 29, no. 6, pp. 647-653, 2011.

[48] M. J. Barry, M. A. Delorenzo, E. S. Walker-Corkery, F. L. Lucas, and D. C. Wennberg, "The rising prevalence of androgen deprivation among older American men since the advent of prostate-specific antigen testing: a population-based cohort study," BJU International, vol. 98, no. 5, pp. 973-978, 2006.

[49] S. L. Chang, J. C. Liao, and R. Shinghal, "Decreasing use of luteinizing hormone-releasing hormone agonists in the United States is independent of reimbursement changes: a medicare and veterans health administration claims analysis," Journal of Urology, vol. 182, no. 1, pp. 255-261, 2009.

[50] G. Lu-Yao, T. A. Stukel, and S. L. Yao, "Changing patterns in competing causes of death in men with prostate cancer: a population based study," Journal of Urology, vol. 171, no. 6, pp. 2285-2290, 2004.

[51] F. Dockery, C. J. Bulpitt, S. Agarwal, M. Donaldson, and C. Rajkumar, "Testosterone suppression in men with prostate cancer leads to an increase in arterial stiffness and hyperinsulinaemia," Clinical Science, vol. 104, no. 2, pp. 195-201, 2003.

[52] M. R. Smith, H. Lee, and D. M. Nathan, "Insulin sensitivity during combined androgen blockade for prostate cancer," Journal of Clinical Endocrinology and Metabolism, vol. 91, no. 4, pp. 1305-1308, 2006.

[53] N. L. Keating, A. J. O’Malley, and M. R. Smith, "Diabetes and cardiovascular disease during androgen deprivation therapy for prostate cancer," Journal of Clinical Oncology, vol. 24, no. 27, pp. 4448-4456, 2006.

[54] S. M. H. Alibhai, M. Duong-Hua, R. Sutradhar et al., "Impact of androgen deprivation therapy on cardiovascular disease and diabetes," Journal of Clinical Oncology, vol. 27, no. 21, pp. 3452-3458, 2009.

[55] M. J. Lage, B. L. Barber, and R. A. Markus, "Association between androgen-deprivation therapy and incidence of diabetes among males with prostate cancer," Urology, vol. 70, no. 6, pp. 1104-1108, 2007.

[56] J. P. Nobes, S. E. Langley, T. Klopper, D. Russell-Jones, and R. W. Laing, "A prospective, randomized pilot study evaluating the effects of metformin and lifestyle intervention on patients with prostate cancer receiving androgen deprivation therapy," BJU International, vol. 109, no. 10, pp. 1495-1502, 2012.

[57] T. Lebret, P. Coloby, J. L. Descotes, S. Droupy, M. Geraud, and B. Tombal, "Educational tool-kit on diet and exercise: survey of prostate cancer patients about to receive androgen deprivation therapy," Urology, vol. 76, no. 6, pp. 1434-1439, 2010.

[58] E. Isahaya, N. Hara, T. Nishiyama, T. Hoshii, I. Takizawa, and K. Takahashi, "Bone metabolic disorder in patients with prostate cancer receiving androgen deprivation therapy (ADT): impact of ADT on the growth hormone/insulin-like growth factor-I/parathyroid hormone axis," Prostate, vol. 70, no. 2, pp. 155-161, 2010.

[59] N. Hara, T. Nishiyama, I. Takizawa, T. Saito, Y. Kitamura, and K. Takahashi, "Decline of the red blood cell count in patients receiving androgen deprivation therapy for localized prostate cancer: impact of ADT on insulin-like growth factor-1 and erythropoiesis," Urology, vol. 75, no. 6, pp. 1441-1445, 2010.

[60] N. Hara, I. Takizawa, E. Isahaya et al., "Insulin-like growth factor-1 is associated with regulation of the luteinizing hormone production in men receiving androgen deprivation therapy with gonadotropin-releasing hormone analogues for localized prostate cancer," Urologic Oncology. In press.

[61] F. Ishizaki, N. Hara, I. Takizawa et al., "Deficiency in androgens and upregulation of insulin-like growth factor-1 are involved in high bone turnover in men receiving androgen deprivation therapy for prostate cancer," Growth Hormone \& IGF Research. In press.

[62] R. J. Dennis, D. Maldonado, M. X. Rojas et al., "Inadequate glucose control in type 2 diabetes is associated with impaired lung function and systemic inflammation: a cross-sectional study," BMC Pulmonary Medicine, vol. 10, article 38, 2010.

[63] K. Omori, T. Ohira, Y. Uchida et al., "Priming of neutrophil oxidative burst in diabetes requires preassembly of the NADPH oxidase," Journal of Leukocyte Biology, vol. 84, no. 1, pp. 292-301, 2008.

[64] T. You, B. J. Nicklas, J. Ding et al., "The metabolic syndrome is associated with circulating adipokines in older adults across a wide range of adiposity," Journals of Gerontology, vol. 63, no. 4, pp. 414-419, 2008.

[65] D. C. Lieb, H. K. Parson, G. Mamikunian, and A. I. Vinik, "Cardiac autonomic imbalance in newly diagnosed and established diabetes is associated with markers of adipose tissue inflammation," Experimental Diabetes Research, vol. 2012, Article ID 878760, 8 pages, 2012.

[66] P. R. Kramer, S. F. Kramer, and G. Guan, “ $17 \beta$-estradiol regulates cytokine release through modulation of CD16 expression in monocytes and monocyte-derived macrophages," Arthritis and Rheumatism, vol. 50, no. 6, pp. 1967-1975, 2004.

[67] G. D. Norata, G. Tibolla, P. M. Seccomandi, A. Poletti, and A. L. Catapano, "Dihydrotestosterone decreases tumor necrosis factor- $\alpha$ and lipopolysaccharide-induced inflammatory response in human endothelial cells," Journal of Clinical Endocrinology and Metabolism, vol. 91, no. 2, pp. 546-554, 2006.

[68] D. Kapoor, S. Clarke, R. Stanworth, K. S. Channer, and T. H. Jones, "The effect of testosterone replacement theraphy on adipocytokines and C-reactive protein in hypogonadal men with type 2 diabetes," European Journal of Endocrinology, vol. 156, no. 5, pp. 595-602, 2007.

[69] S. Y. Kalinchenko, Y. A. Tishova, G. J. Mskhalaya, L. J. G. Gooren, E. J. Giltay, and F. Saad, "Effects of testosterone 
supplementation on markers of the metabolic syndrome and inflammation in hypogonadal men with the metabolic syndrome: the double-blinded placebo-controlled Moscow study," Clinical Endocrinology, vol. 73, no. 5, pp. 602-612, 2010.

[70] E. J. Hamilton, E. Gianatti, B. J. Strauss et al., "Increase in visceral and subcutaneous abdominal fat in men with prostate cancer treated with androgen deprivation therapy," Clinical Endocrinology, vol. 74, no. 3, pp. 377-383, 2011.

[71] M. M. Thewissen, J. G. Damoiseaux, A. M. Duijvestijn et al., "Abdominal fat mass is associated with adaptive immune activation: the CODAM study," Obesity, vol. 19, no. 8, pp. 1690-1698, 2011.

[72] S. Komatsu, N. Hara, F. Ishizaki et al., "Altered association of interleukin-6 with sex steroids in lipid metabolism disorder in men with prostate cancer receiving androgen deprivation therapy," The Prostate. In press. 


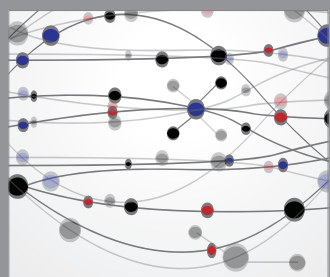

The Scientific World Journal
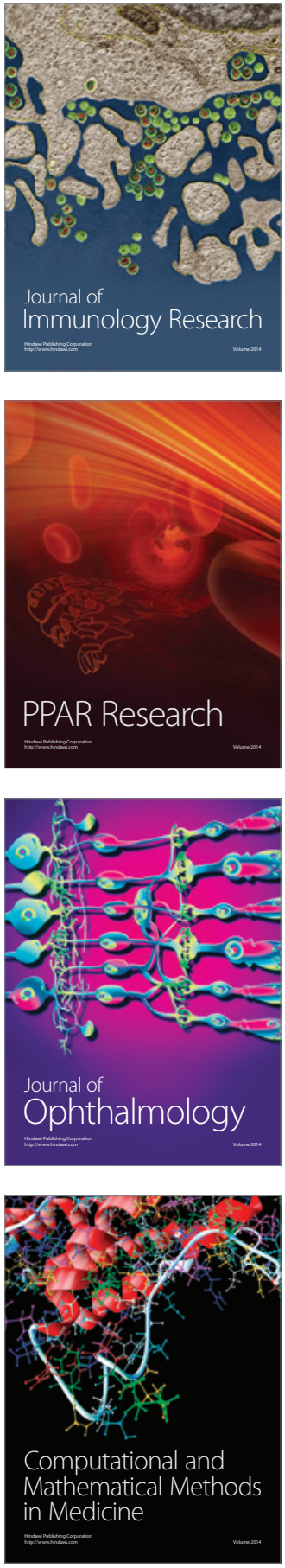

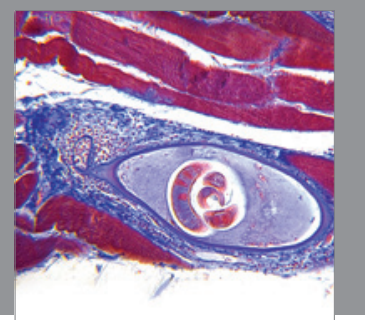

Gastroenterology

Research and Practice
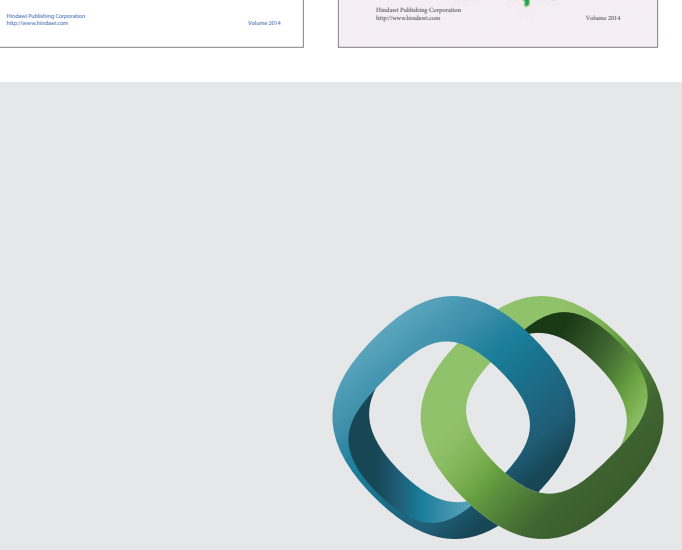

\section{Hindawi}

Submit your manuscripts at

http://www.hindawi.com
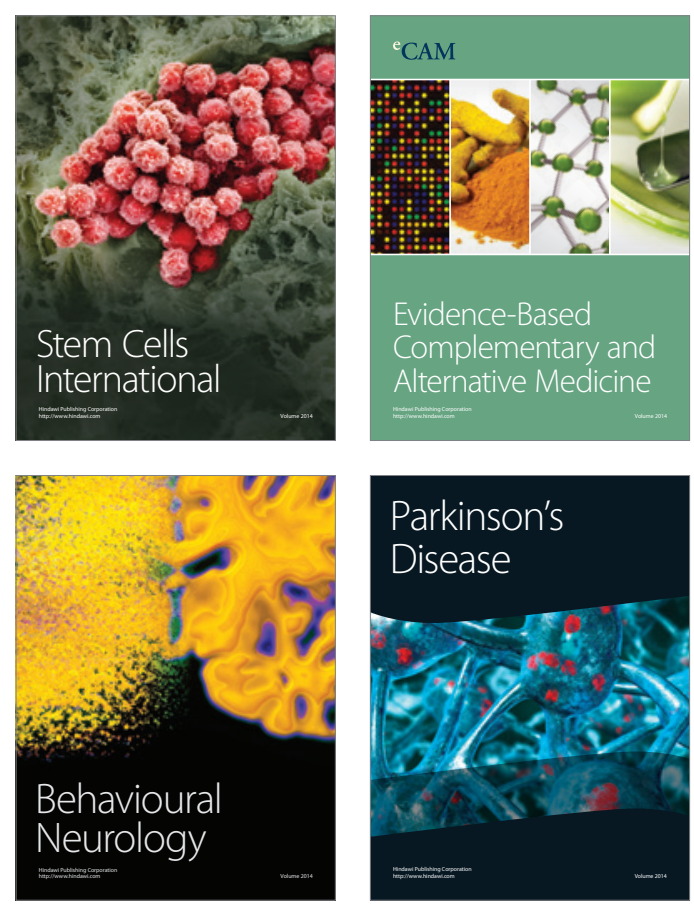

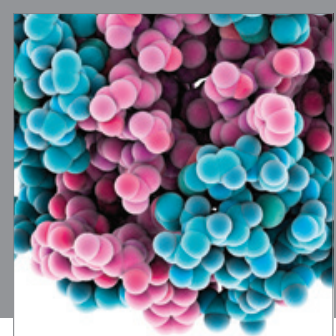

Journal of
Diabetes Research

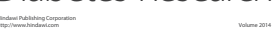

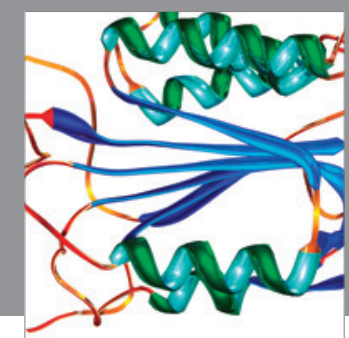

Disease Markers
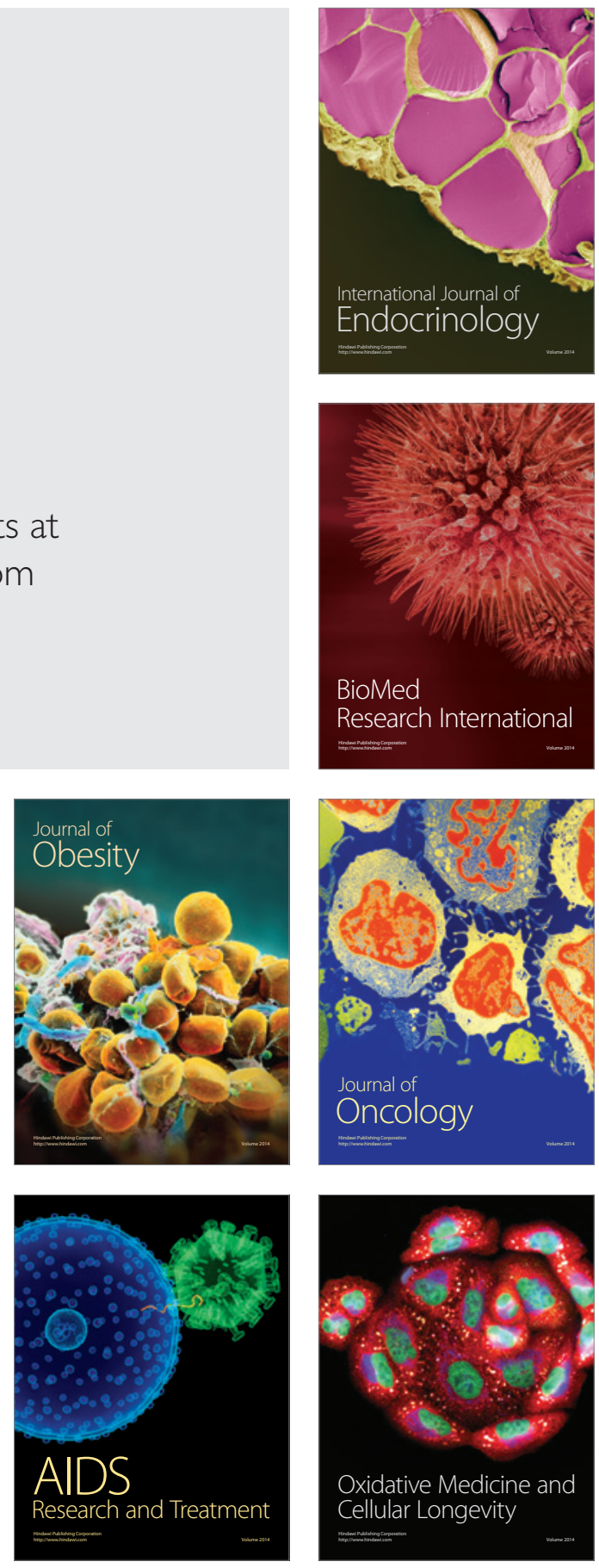